\title{
Comparing the effect on the AGS longitudinal emittance of gold ions from the BtA stripping foil with and without a Booster Bunch Merge
}

K. Zeno

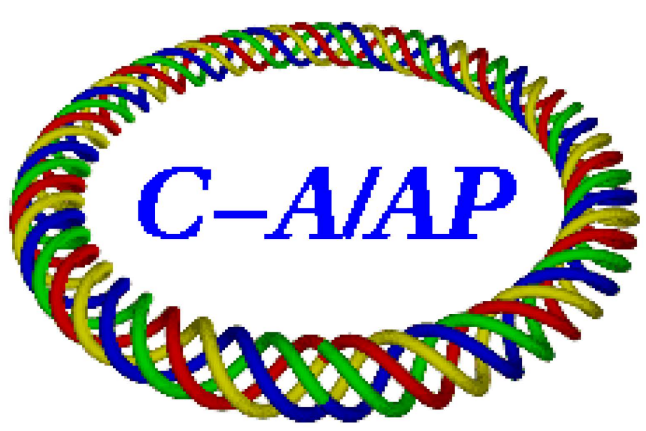

Collider-Accelerator Department Brookhaven National Laboratory Upton, N.Y. 11973

\section{U.S. Department of Energy Office of Science, Office of Nuclear Physics}

Notice: This document has been authorized by employees of Brookhaven Science Associates, LLC under Contract No. DE-SC0012704 with the U.S. Department of Energy. The United States Government retains a non- exclusive, paid-up, irrevocable, world-wide license to publish or reproduce the published form of this document, or allow others to do so, for United States Government purposes. 


\section{DISCLAIMER}

This report was prepared as an account of work sponsored by an agency of the United States Government. Neither the United States Government nor any agency thereof, nor any of their employees, nor any of their contractors, subcontractors, or their employees, makes any warranty, express or implied, or assumes any legal liability or responsibility for the accuracy, completeness, or any third party's use or the results of such use of any information, apparatus, product, or process disclosed, or represents that its use would not infringe privately owned rights. Reference herein to any specific commercial product, process, or service by trade name, trademark, manufacturer, or otherwise, does not necessarily constitute or imply its endorsement, recommendation, or favoring by the United States Government or any agency thereof or its contractors or subcontractors. The views and opinions of authors expressed herein do not necessarily state or reflect those of the United States Government or any agency thereof. 
Comparing the Effect on the AGS Longitudinal Emittance of Gold Ions from the BtA Stripping Foil with and without a Booster Bunch Merge

Keith Zeno

$12 / 18 / 2017$ 
The aim of this note to better understand the effect of merging the Gold bunches in the Booster into one on the resulting AGS longitudinal emittance as compared to not merging them. The reason it matters whether they are merged or not is because they pass through a stripping foil in the BtA line. Data was taken last run (Run 17) for the case where the bunches are not merged, and it will be compared with data from cases where the bunches are merged. Previous data from Tandem operation will also be considered.

There are two main pieces to this puzzle. The first is the $\varepsilon$ growth associated with the energy spread due to 'energy straggling' in the BtA stripping foil and the second is the effective $\varepsilon$ growth associated with the energy loss that occurs while passing through the foil. Both of these effects depend on whether or not the Booster bunches have been merged into one.

\section{Emittance Growth from Energy Straggling in the BtA Foil}

It is thought that the stripping foil causes an energy spread mainly through a mechanism known as 'energy straggling'. Energy straggling is an unavoidable fluctuation in the energy loss associated with the statistical nature of the collisions that occur with the electrons and nuclei in the foil material. ${ }^{1}$ The foil's contribution to the $\Delta E$ of the beam emerging from the foil depends on the characteristics of the foil and incident beam (ex.-energy and charge), but it is likely largely independent of the incident beam's $\Delta E$. So, it seems reasonable then to add the incident beam's $\Delta E$ and the contribution to $\Delta E$ from the foil in quadrature to find the $\Delta E$ of the emerging bunch. That is,

$$
\Delta E_{a}(t)^{2}=\Delta E_{b}(t)^{2}+\Delta E_{f}^{2}
$$

where $\Delta E_{a}(t), \Delta E_{b}(t)$, and $\Delta E_{f}$ are the energy spread half widths after, before and due to the foil, respectively, as a function of the time, $t$, from to the center of the bunch. It's assumed here that $\Delta E_{f}$ is not affected by the bunch passing through the foil (ex.- by heating it over the short amount of time the bunch takes to pass through it), and so it is not treated as a function of $t$. Assuming the bunch in phase space when exiting the Booster has a maximum $\Delta E$ at $t=0, \Delta E_{a}(0)$ and $\Delta E_{b}(0)$ will be the maximum energy spreads simply denoted as $\Delta E_{a}$ and $\Delta E_{b}$. So, the smaller the $\Delta E$ of the incoming bunches the greater the contribution straggling in the foil has to the $\Delta E$ of the beam exiting it. Although other factors are important, for a given total $\varepsilon$ divided between $n$ bunches, the greater $n$ is, the greater the $\varepsilon$ growth due to the foil.

\footnotetext{
${ }^{1}$ P. Thieberger et al, "Improved gold ion stripping at 0.1 and $10 \mathrm{GeV} /$ nucleon for the Relativistic Heavy lon Collider" Phys. Rev. Spec Topics, 2008, pg. 011001-8. There may also be some contribution to the energy spread due to the variation in the energy loss at different locations on the foil as a result of surface irregularities, P. Thieberger, private communication.
} 


\section{Emittance Growth without a Booster Merge}

The foils used are composed of a combination of Aluminum and Carbon. Two foils of this type have been loaded into the BtA foil selector since 2007 and they have been used since then for Gold operation. They are used interchangeably because there is no discernable difference in their performance. In 2007, when no Booster merge was used and there were 6 bunches of Tandem beam per cycle transferred to the AGS, $\Delta E_{b}$ was $18 \mathrm{MeV}, \Delta E_{a}$ was $32 \mathrm{MeV}$, and the total $\varepsilon$ of the 6 bunches at Booster extraction was $0.046 \mathrm{eV}-\mathrm{s} / \mathrm{n} .^{2} \Delta E_{a}$ was found from a debunching measurement in the AGS. ${ }^{3}$ Using eq. (1) and these values for $\Delta E_{b}$ and $\Delta E_{a}$, a value of $26.5 \mathrm{MeV}$ is obtained for $\Delta E_{f}$.

Assuming the shape in phase space of the bunch at Booster extraction is elliptical, which is a reasonable assumption because the bucket was much larger than the bunch, its $\varepsilon$ (per nucleon) would be given by,

$$
\varepsilon=\frac{1}{197} \cdot \pi \cdot \Delta \mathrm{E} \cdot \frac{l}{2}
$$

where $l$ is the bunch length, $\Delta E=\Delta E_{b}$, and 197 is the number of nucleons in a Gold atom. Using this approximation, the $\varepsilon$ of the 6 bunches is $0.0474 \mathrm{eV}-\mathrm{s} / \mathrm{n}$ (the measured bunch length, $l$, was 55 ns). But once the bunch has passed through the foil it is no longer elliptical because the foil's effect is to change $\Delta E_{a}(t)$ according to eq. (1).

Using this approximation, before passing through the foil the curve that traces the outside of the bunch is given by the equation of an ellipse,

$$
\frac{t^{2}}{(l / 2)^{2}}+\frac{\Delta E_{b}(t)^{2}}{\Delta E_{b}^{2}}=1
$$

Using this and eq. (1) it follows that the curve that traces the outside of the bunch after it has passed through the foil will be given by,

$$
\Delta E_{a}(t)^{2}=\Delta E_{b}^{2}\left(1-\frac{t^{2}}{(l / 2)^{2}}\right)+\Delta E_{f}^{2}
$$

\footnotetext{
${ }^{2}$ L. Ahrens, et al, "Setup and Performance of the RHIC Injector Accelerators for the 2007 Run with Gold lons". Proceedings of PACO7, Albuquerque, New Mexico, USA

${ }^{3} \mathrm{~L}$. Ahrens, et al, "Setup and Performance of the RHIC Injector Accelerators for the 2007 Run with Gold lons". Proceedings of PAC07, Albuquerque, New Mexico, USA and P. Thieberger et al, "Improved gold ion stripping at 0.1 and $10 \mathrm{GeV} /$ nucleon for the Relativistic Heavy Ion Collider" Phys. Rev. Spec Topics, 2008 . In the first reference it is noted that the $\varepsilon_{\text {total }}$ of $0.082 \mathrm{eV}-\mathrm{s} / \mathrm{n}$ in the AGS is found from the measured bunch length (55 ns) in matched buckets. Presumably, the buckets are just assumed to be matched because the bunches have a height of $32 \mathrm{MeV}$, and $(32 / 18)(0.046)=0.082$.
} 
Figure 1 shows a plot of this curve together with the ellipse described by eq. (3) and an ellipse with the same $\Delta E_{a}$ as was measured in the AGS (32 MeV). Not surprisingly, the curve generated by eq. (4) does not look like an ellipse at all.

Using numerical integration one finds that the area inside the eq. (4) curve is $0.1013 \mathrm{eV}-\mathrm{s} / \mathrm{n}$, so the $\varepsilon$ has grown by a factor of $0.1013 / 0.0474=2.14$. But this is not the whole story because the bunch will filament out to occupy an area even larger than this. However, the beam initially contained within an ellipse with $\Delta E=32 \mathrm{MeV}$ and $l=55$ ns could be matched to an AGS Rf bucket and would have an $\varepsilon$ of $0.0842 \mathrm{eV}-\mathrm{s} / \mathrm{n}$. So, the $\varepsilon$ growth of that portion of the beam would be $(0.0842 / 0.0474)=1.78$, which is the same as the ratio $\Delta E_{a} / \Delta E_{b}$ since the ellipses have the same length. For simplicity, I'll ignore the beam in the part of the region described by eq. (4) that is outside the $55 \mathrm{~ns}-32 \mathrm{MeV}$ ellipse and consider the $\varepsilon$ growth to be just 1.78 .

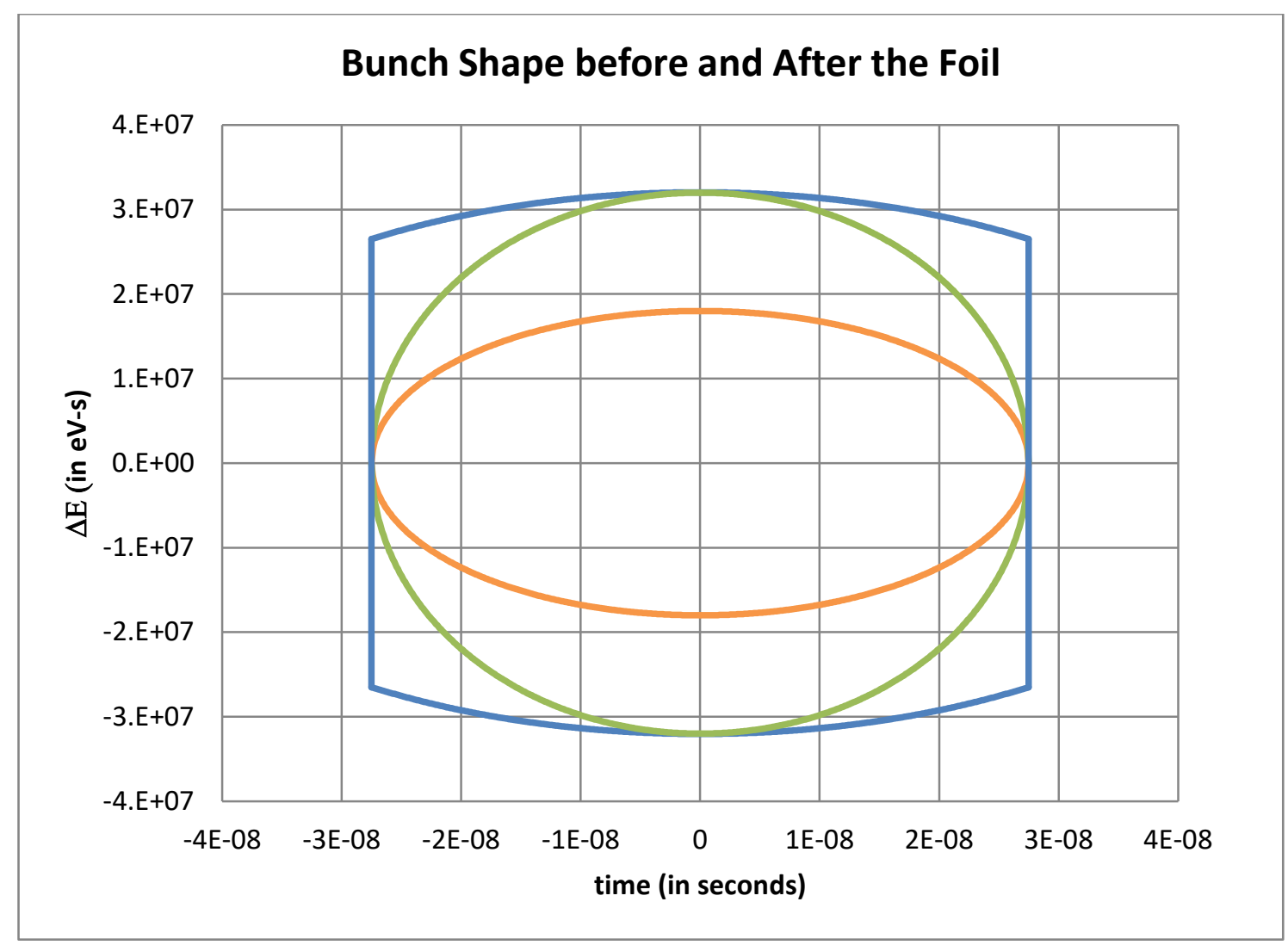

Figure 1: Shapes of the particle distributions in longitudinal phase space at Booster extraction (18 MeV $\Delta \mathrm{E}$ and $55 \mathrm{~ns}$ length, in orange) and AGS injection (32 MeV $\Delta \mathrm{E}$ and $55 \mathrm{~ns}$ length, in blue). Also shown is an ellipse with the same maximum $\triangle \mathrm{E}$ and length as the AGS distribution (in green).

In 2017 a similar measurement was performed for EBIS Au bunches without a Booster merge. The minimum Rf frequency available sets the minimum number of bunches in the Booster (before any merge is performed). In the EBIS case the injection energy is higher (2.0 vs. $0.9 \mathrm{MeV} / \mathrm{n}$ ) so there are 4 bunches instead of 6 . 
The average length (as measured on the first turn in the AGS on the wall current monitor) of the 6 bunches was $79.1 \mathrm{~ns}$ and the $\sigma$ was $3.1 \mathrm{~ns}$. The Rf voltage at Booster extraction estimated from a synchrotron frequency measurement is $26.2 \mathrm{kV}$ and extraction occurs near the peak of the cycle where $\mathrm{dB} / \mathrm{dt}$ near zero. ${ }^{4}$ From this, using Bbat, the $\varepsilon$ of the 4 bunches $\left(\varepsilon_{\text {total }}\right)$ at Booster extraction is $0.068 \pm 0.005 \mathrm{eV}-\mathrm{s} / \mathrm{n}$ and $\Delta E_{b}$ is $27.0 \pm 1.0 \mathrm{MeV}{ }^{5}$

$\Delta E_{a}$ at AGS injection was found by adjusting the $\mathrm{h}=24 \mathrm{Rf}$ voltage to match the $\Delta E$ of the incoming bunches by minimizing bunch shape (quadrupole) oscillations and then measuring the synchrotron frequency ( $3.1 \mathrm{kHz})$ to find the Rf voltage $(61 \mathrm{kV})$. This corresponds to a $\Delta E_{a}$ of $36.7 \pm 1.3 \mathrm{MeV}$. Using the same bunch length found for the Booster extraction measurement (79.1 $\pm 3.1 \mathrm{~ns}$ ) and again assuming an elliptical distribution, a value for $\varepsilon_{\text {total }}$ of $0.092 \pm 0.07 \mathrm{eV}-\mathrm{s} / \mathrm{n}$ is obtained using eq. (2). ${ }^{6}$ So, in the EBIS case $\Delta E$ grows by a factor of $1.36 \pm 0.15$. Note that this is less than the factor of 1.78 in the Tandem case. Using eq. (1) the value of $\Delta E_{f}$ is $24.9 \pm 0.9$ vs. 26.5 $\mathrm{MeV}$ found for Tandem beam. ${ }^{7}$

In 2014 the data for an emittance measurement at extraction without a Booster merge was recorded. In this case four measurements are used to obtain a length of $90.4 \pm 2.8 \mathrm{~ns}$. The $\mathrm{Rf}$ voltage obtained from the synchrotron frequency was $25.2 \mathrm{kV}$. ${ }^{8}$ This gives an $\varepsilon_{\text {total }}$ at Booster extraction of $0.084 \pm 0.005 \mathrm{eV}-\mathrm{s} / \mathrm{n}$. This is quite a bit larger than the value obtained in Run 17 $(0.068 \pm 0.005 \mathrm{eV}-\mathrm{s} / \mathrm{n})$.

Figure 2 shows a couple of the bunches from Run 14 and 17 on the first turn in AGS. Inspection shows that the bunches are somewhat smaller in Run 17 (top), the Rf voltage measured from the synchrotron frequency is about the same as in the Run 17 case, 26.2 vs. 25.2 $\mathrm{kV}$. A current transformer trace was dumped into the elog around the time of this measurement and it indicates about a $4 \%$ loss at the end of the merge porch. ${ }^{9}$ A similar picture from Run 14 also shows about a $4 \%$ loss there. ${ }^{10}$ So, it seems unlikely that the difference in lengths is due to more (unintentional) longitudinal scraping in the 2017 case. So, it seems that the $\varepsilon$, at least up

\footnotetext{
${ }^{4}$ The synchrotron frequency was measured with merged bunches. It is the value obtained in the "Emittance Growth with Merged Bunches" section below (corresponding to $21.31 \mathrm{kV}$ ) and was measured over the last ms before extraction for a setting of $26 \mathrm{kV}$. The setting here was $32 \mathrm{kV}$. The expected voltage then is $(32 / 26) * 21.31 \mathrm{kV}=26.2 \mathrm{kV}$, which is $82 \%$ of the reference.

${ }^{5}$ Booster-AGS-EBIS Jun 202017 elog 1450 entry. Although I've changed the bunch length and voltage after more careful measurements were made. Bbat is an application used extensively in this note to calculate emittances, bucket areas, energy spreads, and the like.

${ }^{6}$ Booster-AGS-EBIS Jun 192017 elog 1617 entry, again though the bunch length used here is different.

7 The extraction energy is slightly higher for EBIS than it is for Tandem (103.9 vs. $103.2 \mathrm{MeV} / \mathrm{n}$ ). Also, the Tandem beam was Au31+ and EBIS provides Au32+. Both of these differences are treated as negligible.

${ }^{8}$ See Booster-AGS-EBIS Jun 2420141652 to 1725 entries. A more careful bunch length measurement was made than the one in the elog and Bbat was used to calculate the $\varepsilon$, the ellipse approximation would give $0.085 \mathrm{eV}-\mathrm{s} / \mathrm{n}$. The measured bunch length shown in the elog was $88.3 \mathrm{~ns}$.

${ }^{9}$ Booster-AGS-EBIS Jun 1620171657 entry

${ }^{10}$ Booster-AGS-EBIS Jun 2420141655 entry
} 
until the merge porch, in Run 17 is only about $81 \%$ of what it was in Run 14 . There is no AGS data for the case where there was not a Booster merge for Run 14.

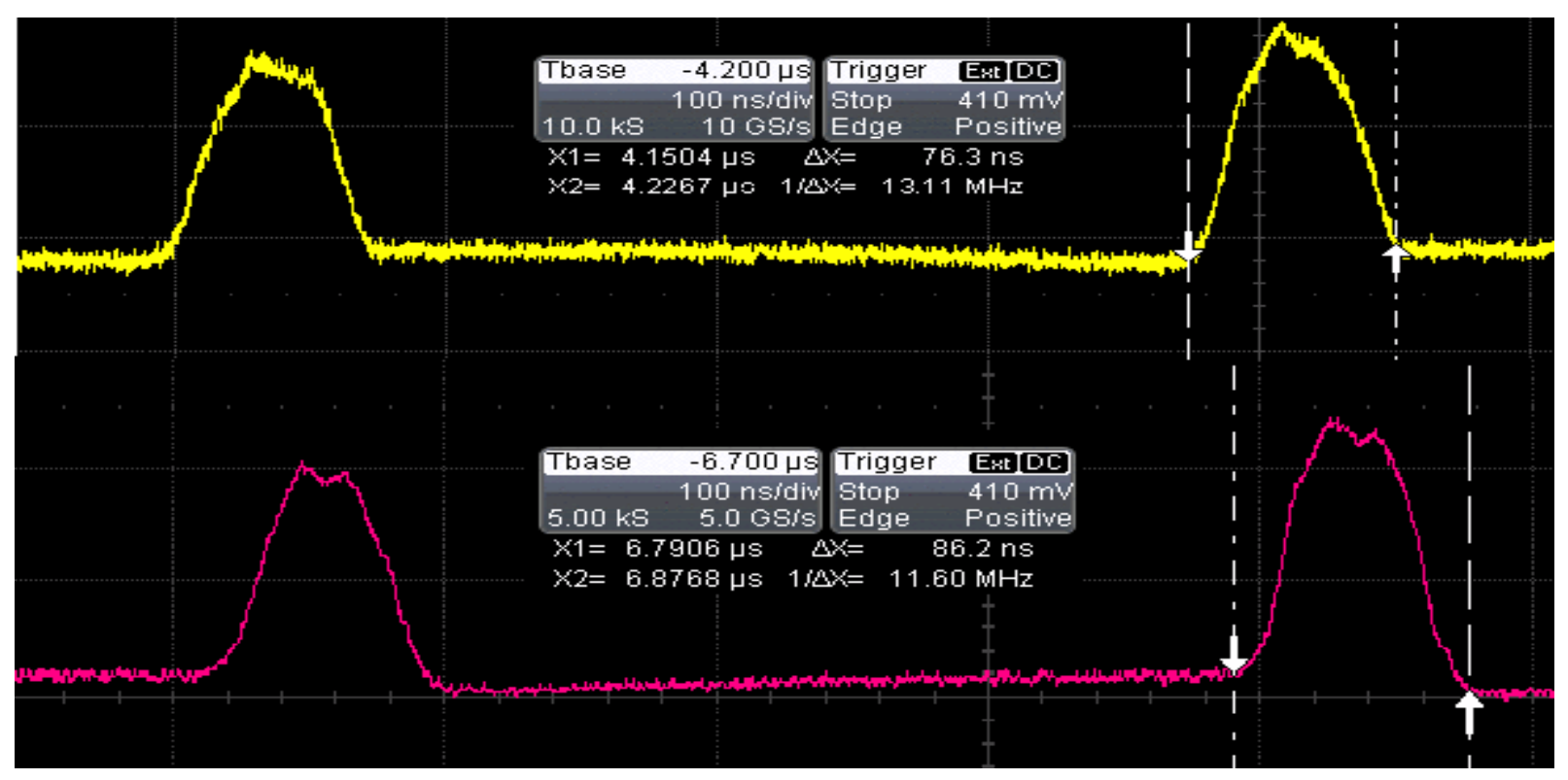

Figure 2: Bunches without a Booster merge on the first turn in the AGS as viewed on the WCM. The top trace is from Run 17 and the bottom is from Run $14 .{ }^{11}$

\section{Emittance Growth with Merged Bunches}

Measuring the $\varepsilon$ of the merged bunch is fraught with difficulties because the bunch extracted from the Booster has a lot of structure and so it is not reasonable to treat it as though it is matched to the bucket. Also, whatever area it occupies in phase space, which is technically its $\varepsilon$, will be smaller than the area (enclosed by a simple curve) that it will eventually occupy after filamentation has occurred.

Although the bunch has a lot of structure, the envelope of the Booster Wall Current Monitor (WCM) near extraction only shows a maximum variation in height of about 6\% (see Figure 3). However, the actual variation may be larger because the time response of the Booster WCM is not sufficient to show spikes in the distribution. ${ }^{12}$ Figure 3 also shows how a typical bunch appears on the first turn in the AGS on the WCM (which does have a sufficient time response).

\footnotetext{
${ }^{11}$ The top trace is from Booster-AGS-EBIS Jun 1620171709 entry and the bottom one is from Booster-AGS-EBIS Jun 2420141652 entry.

${ }^{12}$ See for example Figure 9 in K. Zeno, "Longitudinal Emittance Measurements in the Booster and AGS during the 2014 RHIC Gold Run", C-A/AP/523, August 2014.
} 
A Booster extraction $\varepsilon$ measurement was made in 2016 using an Rf voltage of $21.31 \mathrm{kV}$, obtained from the synchrotron frequency during the last ms before extraction $(944 \mathrm{~Hz}){ }^{13}$ Thirteen bunch length measurements were also made on the first turn in the AGS and their average value was 241.5 ns with a $\sigma$ of 17 ns. For a matched bunch this gives an $\varepsilon$ of $0.072 \pm 0.010 \mathrm{eV}-\mathrm{s} / \mathrm{n}$, as compared to $0.068 \pm 0.005 \mathrm{eV}-\mathrm{s} / \mathrm{n}$ for unmerged bunches.

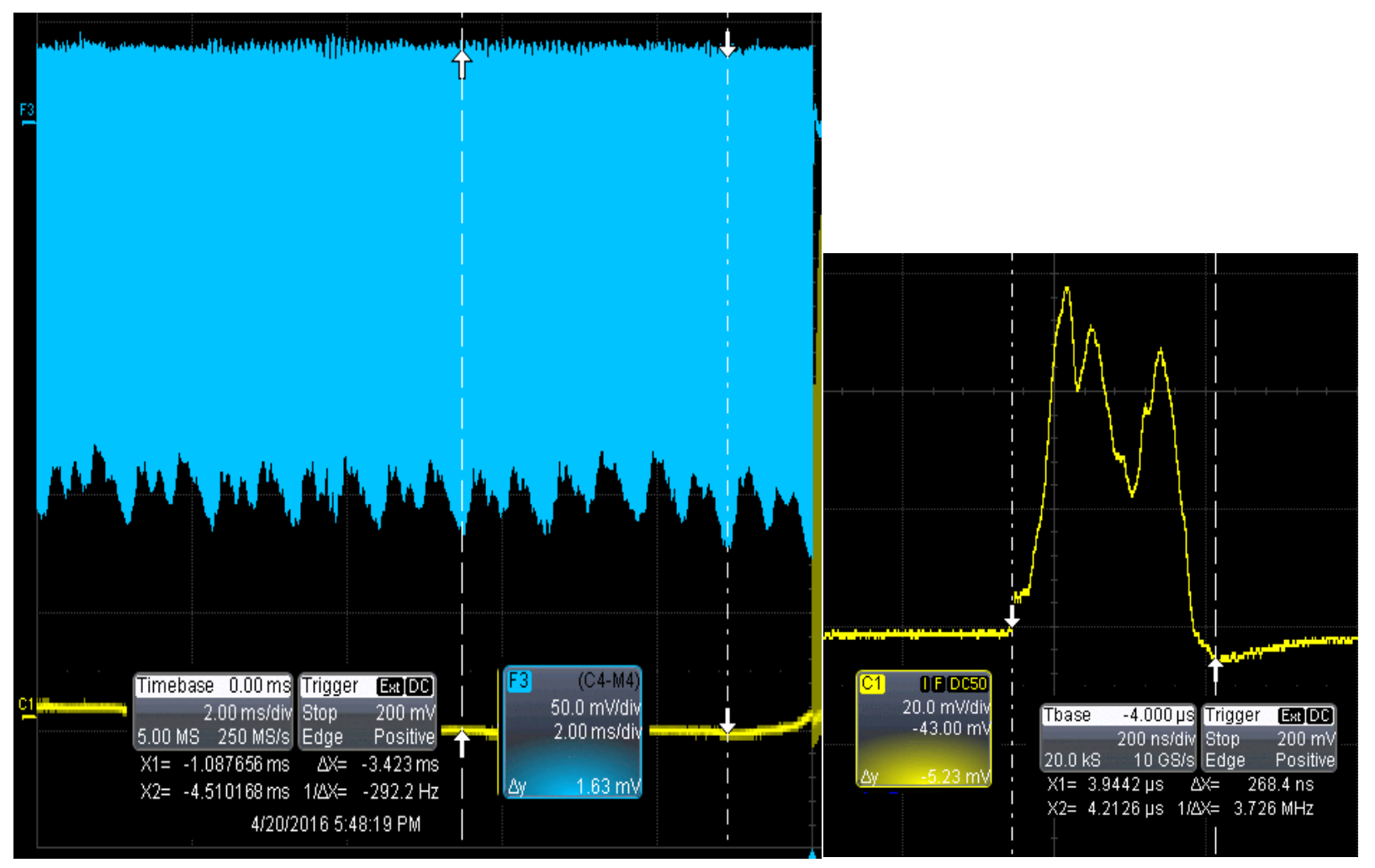

Figure 3: The Booster WCM during the last $10 \mathrm{~ms}$ of the cycle with a merged bunch (left) and a merged bunch on the first turn in the AGS as seen on the WCM (right). ${ }^{14}$

For this measurement, the voltage is at its maximum amplitude after the merge, but then drops, until 3 ms before extraction where its reference corresponds to $21.31 \mathrm{kV}$, where it remains until extraction. The voltage was likely set about as low as possible before beam loss starts to occur, which would then correspond to a bucket the area of which is about the same as the bunch's $\varepsilon .{ }^{15}$ The $\mathrm{dB} / \mathrm{dt} 3 \mathrm{~ms}$ before extraction, or at least its set value, is $68.3 \mathrm{~g} / \mathrm{ms}$ and it remains there for another $1.2 \mathrm{~ms}$ at which time it drops rapidly and is zero by the time beam is extracted. The bucket area, calculated using the expected $\mathrm{B}$ field and $\mathrm{dB} / \mathrm{dt}$ from the main magnet function is $0.069 \mathrm{eV}$-s/n just before $\mathrm{dB} / \mathrm{dt}$ begins to drop, but only a small change in either $\mathrm{dB} / \mathrm{dt}$ or voltage is required for it to reach $0.072 \mathrm{eV}-\mathrm{s} / \mathrm{n}$. For example, lowering $\mathrm{dB} / \mathrm{dt}$ from 68.3 to 68.0

\footnotetext{
${ }^{13}$ Booster-AGS-EBIS Apr 202015 elog

${ }_{14}$ Booster-AGS-EBIS Apr 202016 elog 1750 entry (left) and Booster-AGS-EBIS Jun 1620161650 entry (right)

15 It was lowered to this level to accommodate quad mode pumping (which was turned off for these measurements).
} 
$\mathrm{g} / \mathrm{ms}$ or raising the Rf voltage from 21.31 to $21.39 \mathrm{kV}$ is enough to increase the bucket area to $0.072 \mathrm{eV}-\mathrm{s} / \mathrm{n}$. Both of these changes fall well within the range of possible actual values for these 2 parameters. ${ }^{16}$ So, although the $\varepsilon$ measurement made at Booster extraction assumes a matched bunch, the value obtained by that method is not inconsistent with the possible values for the $\varepsilon$ of a bunch (matched or unmatched) that could pass through this bucket area minimum.

\section{Debunching Measurements of Merged Bunches at AGS Injection}

So, now what can be said of the AGS $\varepsilon$ measurement of the merged bunch? There are 2 aspects to it; the first is its $\varepsilon$ right at injection which can be estimated by finding the maximum $\Delta p / p$ of the incoming bunch by measuring the time it takes to debunch. That together with the bunch length can be used to define an ellipse (according to eq. (2)) which has a certain area which then can be used, at least as a first approximation, for the bunch's $\varepsilon$. Even though the injected bunch can not be matched to an AGS bucket, the measurement of its debunching time should still provide the maximum extent of the bunch's $\Delta p / p$ provided that the maximum and minimum $\Delta p / p$ are roughly of the same magnitude (which can be checked during the measurement). After injection the bunch will filament out in the bucket it's in, and will then be more or less matched, so that its $\varepsilon$ can actually be measured.

Figure 4 shows a debunching measurement from Feb 10, 2016. A bunch is injected with the Rf off and viewed on a mountain range display. The faster particles appear to move the left and the slower ones to the right. Eventually they meet and the time it takes for this to happen, known as the debunching time $\left(t_{d b}\right)$, is related to the extent of the beam's $\Delta p / p$. One estimates by looking at this display when this happens. The topmost highlighted trace is the guess for when it happens. In this case it occurs on the $70^{\text {th }}$ trace which is $3.44 \mathrm{~ms}$ after injection. Note that the distribution of lower momentum particles is roughly similar to the distribution of higher ones.

The relationship between $t_{d b}$ and $\Delta p / p$ comes from the equation that relates the change in the revolution period $(\Delta \tau)$ per revolution for a particle with a momentum deviation of $\Delta p / p$,

$$
\frac{\Delta \tau}{\tau}=\eta \frac{\Delta p}{p}
$$

where $\tau$ is the revolution period of an on-momentum particle and $\eta$ is the slip factor. $\Delta \tau$ is related to the change in the azimuthal angle around the ring per turn $(\Delta \theta)$ of a particle with a momentum deviation of $\Delta p / p$ by,

$$
\Delta \tau=\frac{\Delta \theta}{2 \pi} \tau
$$

\footnotetext{
${ }^{16}$ The bucket area at $3 \mathrm{~ms}$ before extraction with $21.31 \mathrm{kV}$ and $68.3 \mathrm{~g} / \mathrm{ms}$ is also $0.069 \mathrm{eV}-\mathrm{s} / \mathrm{n}$ and similarly small changes to the voltage or $\mathrm{dB} / \mathrm{dt}$ would bring it up to $0.072 \mathrm{eV}-\mathrm{s} / \mathrm{n}$.
} 
The highest $\Delta p / p$ particles will meet up with the ones with the lowest $\Delta p / p$ in $m$ turns so

$$
m \cdot \Delta \theta=\pi-\Delta \phi
$$

where $\Delta \phi$ is the bunch's half-length in terms of azimuthal angle. Since $t_{d b}=m \tau$ it follows that

$$
t_{d b}=\frac{(\pi-\Delta \phi) \tau}{2 \pi \eta \cdot \frac{\Delta p}{p}}
$$

In the case considered here, the measured bunch length at injection was $250 \mathrm{~ns}$, and the $\Delta p / p$ obtained from the $\mathrm{t}_{\mathrm{db}}$ measurement was $1.074 \mathrm{e}-3$, corresponding to a $\Delta E_{a}$ of $42.2 \mathrm{MeV}$.



Figure 4: Mountain range display of the AGS wall current monitor during the first $4 \mathrm{~ms}$ after injection. There are 80 traces and adjacent traces are separated from each other by $50 \mu$ s. Time flows from bottom to top. The highlighted trace in the middle of the display is of the $70^{\text {th }}$ trace with a higher vertical gain and where the $70^{\text {th }}$ trace actually occurs within the 80 traces is shown in the other highlighted trace. The $70^{\text {th }}$ trace, corresponding to $3.44 \mathrm{~ms}$ after injection, is where the two sides of the $\Delta \mathrm{p} / \mathrm{p}$ distribution begin to overlap. The sweep speed is $2 \mu \mathrm{s} / \mathrm{div}$.

In order to estimate how much $\Delta E$ increases because of the foil, there has to be an estimate for $\Delta E_{b}$ at the time of the measurement. Unfortunately, a Booster $\varepsilon$ measurement wasn't made when this debunching measurement was taken. The one taken in April 2016 and discussed above could be used, except that the voltage at Booster extraction is different for the AGS measurement than what it was when the Booster measurement was taken. However, the voltage settings in each case are known, and although the voltage settings do not appear to be calibrated properly the response to different settings is expected to be linear. Assuming this, the Booster voltage when the AGS measurement was taken should be $26.23 \mathrm{kV}$, and using Bbat, a Booster $\varepsilon$ 
of $0.072 \pm 0.01 \mathrm{eV}$-s/n corresponds to a $\Delta E_{b}$ of $39.5 \pm 2.7 \mathrm{MeV}$ (for a matched bunch) so it's estimated that $\Delta E$ changes by about a factor of $42.2 / 39.5=1.07$.

An elliptical bunch with a $\Delta E_{a}$ of $42.2 \mathrm{MeV}$ and a length of $250 \mathrm{~ns}$ is $0.084 \mathrm{eV}-\mathrm{s} / \mathrm{n}$ and $\Delta E_{f}$ obtained from $\Delta E_{a}, \Delta E_{b}$, and eq. (1) is only $14.9 \mathrm{MeV}$. The estimated $\varepsilon$ growth is a factor of $(0.084 / 0.072)=1.17$, which is smaller than the values obtained for EBIS $(1.36 \pm 0.15)$ and Tandem (1.78) bunches without a Booster merge. ${ }^{17}$

Although it may still be reasonable to approximate the smallest envelope that encloses the merged bunch as elliptical in the Booster, when injected into the AGS the matching of an elliptical bunch to the AGS buckets is an issue. The $\mathrm{h}=1$ bucket at extraction in the Booster is about 1520 ns wide, and the buckets that are normally used in the AGS are either $383 \mathrm{~ns}(\mathrm{~h}=16$ ) or $253 \mathrm{~ns}(\mathrm{~h}=24)$ wide. In the $\mathrm{h}=24$ case, the bunch is just about as wide as the bucket, and in the $\mathrm{h}=16$ case the bunch length is still a significant fraction of the bucket length. So, especially in the $\mathrm{h}=24$ case, the paths many of the particles take in phase space will deviate from elliptical. For the 8:4:2 merge employed in the AGS the harmonic used is 16, and for the more recent 12:6:2 merge it is 24 .

Figure 5 shows an overlay of two $250 \mathrm{~ns}$ long bunches with the same $\Delta E(42.2 \mathrm{MeV})$, one is matched to an $\mathrm{h}=16$ bucket and the other to an $\mathrm{h}=24$ one. The $\mathrm{h}=16$ bunch is still nearly elliptical but the $\mathrm{h}=24$ bunch is not. As seen above an elliptical bunch would be $0.084 \mathrm{eV}-\mathrm{s} / \mathrm{n}$, but, using Bbat, the $\varepsilon$ of the $\mathrm{h}=16$ bunch is $0.080 \mathrm{eV}-\mathrm{s} / \mathrm{n}$ and in the $\mathrm{h}=24$ case it is $0.070 \mathrm{eV}-\mathrm{s} / \mathrm{n}$.

For bunches without a Booster merge the $\varepsilon$ when injected into the AGS was approximated as the area of an ellipse with $\Delta E_{a}$ and its half-length as its axes. The justification for this was that whatever beam is injected into this area will not filament out to occupy a greater area, and the density of beam outside that ellipse would be expected to be relatively low. With a merged bunch however, especially for the $h=24$ case, the size and shape of the bucket is less conducive to containing as much of the injected beam. On the other hand, beam that is injected into the $\mathrm{h}=24$ bucket will stay a bit smaller than it would if that beam were injected into a lower harmonic bucket. It's also true that this approximation is worse in the merged case because the bunch's distribution tends to have a larger amount of beam away from its center (see Figure 3).

Two $\varepsilon$ measurements of merged bunches injected into $\mathrm{h}=16$ buckets after filamentation were made during Run 16 and in both cases the measured $\varepsilon$ was in the $0.100-0.115 \mathrm{eV}-\mathrm{s} / \mathrm{n}$

\footnotetext{
${ }^{17}$ The ratio $\Delta \mathrm{E}_{\mathrm{a}} / \Delta \mathrm{E}_{\mathrm{b}}=42.2 / 39.5$ is only 1.07 but the estimated $\varepsilon$ growth is 1.17 because the length of the Booster bunch required to get $0.072 \mathrm{eV}$-s/n with $26.23 \mathrm{kV}$ is $228.9 \mathrm{~ns}$ but the bunch used in the AGS $\Delta p / p$ measurement was $250 \mathrm{~ns}$. That is, the ratio of the ellipse areas is $(250 * 42.2) /(228.9 * 39.5)=1.17$. So, there is some inconsistency here, although a variation in the measured bunch length from cycle to cycle by this amount is not unusual.
} 
range. ${ }^{18}$ Any beam not injected into the buckets would show up as beam loss when the main magnet starts to ramp and there is little or no loss evident there. ${ }^{19}$ The difference between this value for the $\varepsilon$ and the area of an ellipse with $\Delta E_{a}$ and half-length as its axes $(0.084 \mathrm{eV}-\mathrm{s} / \mathrm{n})$ could result from beam that was not initially injected inside of that ellipse and its subsequent filamentation (see Figure 1 and related discussion).

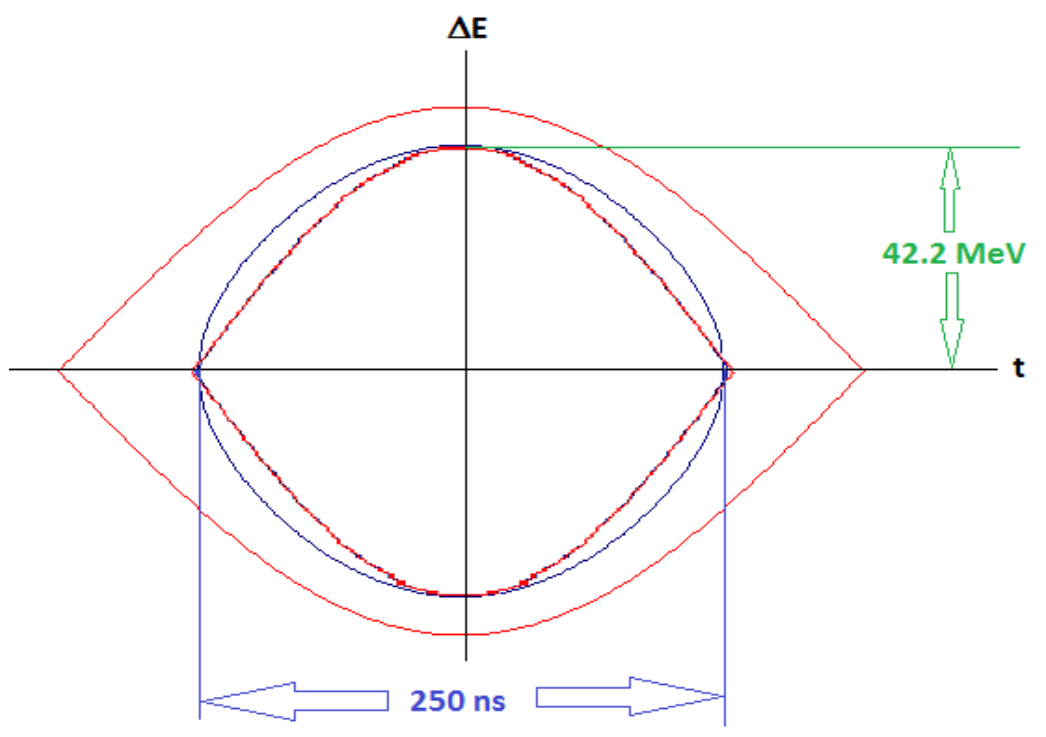

Figure 5: Comparison of two bunches each $250 \mathrm{~ns}$ long and with a $\Delta \mathrm{E}_{\mathrm{a}}$ of $42.2 \mathrm{MeV}$. One is matched to an $\mathrm{h}=16$ bucket and the other to an $\mathrm{h}=24$ bucket at AGS injection energy. The larger red curve is the $h=16$ bucket. The blue curve is the bunch in an $h=16$ bucket. The $h=24$ bunch and bucket overlap except right near the time axis where the bucket is slightly longer (253 vs 250 $n s$ ). The smaller red curve is the $h=24$ bucket, and the $h=24$ bunch (blue) is barely visible because it is essentially the same shape as the bucket.

\section{Debunching Measurements for $h=24$ using Quad Mode Pumping}

$\mathrm{h}=24$ buckets are required at injection for the 12:6:2 merge. But, not only is there an issue with using $\mathrm{h}=24$ in regard to fitting the incoming bunches into the buckets because of their length, but the injection kicker has to have time to rise between when a bunch in the AGS has passed and injection occurs into an adjacent $\mathrm{h}=24$ bucket. ${ }^{20}$ With quad mode pumping at Booster extraction the length of the injected bunches is reduced to allow these two conditions to be satisfied and consequently their $\Delta E$ at Booster extraction increases.

\footnotetext{
${ }^{18}$ See K. Zeno,"Overview and analysis of the 2016 Gold Run in the Booster and AGS", C-A/AP/523, August 2014, pg. 29 and Booster-AGS-EBIS Feb 102016 and Booster-AGS-EBIS Feb 112016 elogs. The range results mainly from whether or not the overshoot on the WCM signal is ignored in the bunch length measurement.

${ }^{19}$ See for example, Booster-AGS-EBIS Feb 8, 2016 elog 1355 entry.

${ }^{20}$ See K. Zeno," Overview and analysis of the 2016 Gold Run in the Booster and AGS", C-A/AP/523, August 2014. Pgs. 13-16 for a discussion of quad pumping.
} 
In Run 16 four debunching measurements were made with bunches that had been quad mode pumped and the results are summarized in Table I. The actual measurements only consist of the bunch length and the debunching time (from which $\Delta p / p$ and $\Delta E_{a}$ are calculated). From the bunch length and $\Delta E_{a}$ the area of the ellipse with those axes is calculated. The $\varepsilon$ of an $\mathrm{h}=24$ bunch (found using Bbat) with those axes is also shown. An estimate for $\Delta E_{b}$ is found by using the measured bunch length and adjusting the Rf voltage to obtain an assumed $\varepsilon$ of $0.072 \mathrm{eV}-\mathrm{s} / \mathrm{n}$ (again using Bbat). The latter was performed even though the Booster $\varepsilon$ was not actually measured for any of these cases.

These measurements were taken over the course of the run. The $\Delta p / p$ in all cases is greater than what was measured without the quad mode pumping and its average is $25 \%$ higher than in that case (1.34 vs.1.07e-3). However, although the largest $\Delta p / p$ is with the largest amount of pumping and the smallest $\Delta p / p$ is with the smallest amount, beyond that the correlation isn't clear.

The average value obtained for $\Delta E_{f}$ from $\Delta E_{a}$ and $\Delta E_{b}$ using eq. (1) is much larger than the one measurement made without pumping (24.6 vs. $14.9 \mathrm{MeV}$ ), and its value for the individual measurements varies by a lot. The average value is however close to the values obtained with unmerged bunches (26.5 MeV for Tandem and 24.9 MeV for EBIS bunches). The average ellipse area found using eq. (2) is similar in both cases $(0.086$ vs. $0.084 \mathrm{eV}-\mathrm{s} / \mathrm{n})$ as is the estimated $\varepsilon$ growth, $0.086 / 0.072=1.19$ vs. 1.17 .

\begin{tabular}{|l|l|l|l|l|l|l|l|l|l|l|}
\hline Date & $\begin{array}{l}\text { Length } \\
(\mathbf{n s})\end{array}$ & $\#$ & $\begin{array}{l}\mathbf{Q P} \\
\mathbf{A m p}\end{array}$ & $\begin{array}{l}\boldsymbol{t}_{\boldsymbol{d} \boldsymbol{b}} \\
(\mathbf{m s})\end{array}$ & $\begin{array}{l}\boldsymbol{\Delta p / p} \\
(\mathbf{e}-3)\end{array}$ & $\begin{array}{l}\Delta \boldsymbol{E}_{\boldsymbol{a}} \\
(\mathbf{M e V})\end{array}$ & $\begin{array}{l}\text { ellipseA } \\
(\mathbf{e V}-\mathbf{s} / \mathbf{n})\end{array}$ & $\begin{array}{l}\mathbf{h = 2 4} \boldsymbol{\varepsilon} \\
(\mathbf{e V}-\mathbf{s} / \mathbf{n})\end{array}$ & $\begin{array}{l}\Delta \boldsymbol{E}_{\boldsymbol{b}} \\
(\mathbf{M e V})\end{array}$ & $\begin{array}{l}\Delta \boldsymbol{E}_{\boldsymbol{f}} \\
(\mathbf{M e V})\end{array}$ \\
\hline Mar 1 & $211 \pm 13$ & 6 & 3.8 & 2.35 & 1.59 & 62.26 & 0.105 & 0.095 & 42.90 & 45.1 \\
\hline Apr 14 & $193 \pm 24$ & 2 & 3.6 & 3.0 & 1.25 & 48.91 & 0.075 & 0.069 & 46.78 & 14.3 \\
\hline Apr 20 & $203.6 \pm 13$ & 7 & 3.6 & 2.69 & 1.39 & 54.79 & 0.089 & 0.081 & 44.40 & 32.1 \\
\hline Jun 16 & $208.2 \pm 3$ & 5 & 2.8 & 3.19 & 1.12 & 43.86 & 0.073 & 0.066 & 43.35 & 6.7 \\
\hline average & 204.0 & - & - & 2.81 & 1.34 & 52.46 & 0.086 & 0.078 & 44.36 & 24.6 \\
\hline$\sigma$ & 7.9 & - & - & 0.32 & 0.17 & 7.9 & 0.015 & 0.013 & 1.5 & 15 \\
\hline
\end{tabular}

Table 1: Summary of debunching measurements made with quad mode pumping on at Booster extraction. The length is the full length as measured on the first turn in the AGS with the wall current monitor and is shown with the standard deviation, the \# column indicates the number of length measurements, QP Amp is the amplitude of the quad mode pumping in $\mathrm{kV}, \mathbf{t}_{\mathbf{d b}}$ is the debunching time, $\Delta \mathbf{p} / \mathbf{p}$ is the half width, and $\Delta \mathbf{E}_{\mathbf{a}}$ is the half width. The ellipse $\mathbf{A}$ (area) is found from eq. (2) and the $\mathbf{h}=\mathbf{2 4} \boldsymbol{\varepsilon}$ is that of a bunch with a maximum $\Delta \mathrm{E}$ of $\Delta \mathrm{E}_{\mathrm{a}}$ with length indicated found using Bbat. $\Delta \mathbf{E}_{\mathbf{b}}$ is the halfwidth at Booster extraction for the length shown found using Bbat with an $\varepsilon$ at Booster extraction of $0.072 \mathrm{eV}-\mathrm{s} / \mathrm{n} . \Delta \mathbf{E}_{\mathrm{f}}$ is calculated from $\Delta \mathrm{E}_{\mathrm{b}}$ and $\Delta \mathrm{E}_{\mathrm{a}}$ using eq. (1). ${ }^{21}$

\footnotetext{
${ }^{21}$ This is from the data in summarized in Table II of K. Zeno," Overview and analysis of the 2016 Gold Run in the Booster and AGS", C-A/AP/523, August 2014. The length in the April 14 case was changed from 180 to 193 ns to better reflect the measurements.
} 


\section{Merged Bunch Emittance Measurements after Filamentation at AGS Injection Energy}

Emittance measurements after filamentation on the AGS injection porch made in Run 16 have been reported before. ${ }^{22}$ The purpose of discussing them again is to see if a better understanding of the results can be found now that the effect of the foil has been studied in more detail. Table II shows the results of the measurements.

The first 2 measurements are for $\mathrm{h}=16$ with no quad mode pumping and the $\varepsilon$ values are somewhat larger than those obtained from debunching measurements ( $\sim 0.103$ to 0.115 vs. $0.086 \mathrm{eV}-\mathrm{s} / \mathrm{n}$ ). This is not surprising because the $\varepsilon$ quoted in the debunching cases is the area of an ellipse which some of the beam is expected to fall outside of, but remain in the bucket, as discussed earlier. The area of an $\mathrm{h}=16$ bucket which matches a bunch to the values found from the debunching measurement $\left(\Delta E_{a}=42.2 \mathrm{MeV}\right.$ and $\left.l=250 \mathrm{~ns}\right)$ is $0.122 \mathrm{eV}-\mathrm{s} / \mathrm{n}$.

The $\mathrm{h}=24$ measurements (with pumping) are significantly smaller than that and are roughly the same as the ellipse area associated with the debunching measurements. Figure 5 shows that a bunch with axes $\Delta E_{a}$ and $l / 2$, at least for the case with no pumping, when injected into an $\mathrm{h}=24$ bucket fills that bucket so that bunch should never get any larger than it. That's not to say that there is no beam that falls outside that bucket, just that it will not be captured into that bunch. Also, those bunches are actually smaller than the ellipse given by eq. (2). But in the cases considered here, the bunches are about the same size as that ellipse.

\begin{tabular}{|l|l|l|l|l|l|l|}
\hline Date & $\begin{array}{l}\text { time from } \\
\text { inj. (ms) }\end{array}$ & $\begin{array}{l}\text { Length } \\
\text { (ns) }\end{array}$ & $\#$ & $\begin{array}{l}\text { Rf V } \\
\text { from S.F. }\end{array}$ & $\begin{array}{l}\text { Bunch } \boldsymbol{\varepsilon} \\
\text { (eV-s/n) }\end{array}$ & Notes \\
\hline Feb 10 & 200 & $258 \pm 5$ & 3 & 30.8 & $0.115 \pm 0.003$ & h=16, No Q.P. \\
\hline Feb 11 & 200 & 271 & 1 & 21.5 & 0.103 & h=16, No Q.P. \\
\hline Apr 14 & 600 & 194 & 1 & 41.3 & 0.085 & h=24, Q.P. \\
\hline Jun 16 & 600 & $195.4 \pm 2.4$ & 5 & 30.5 & $0.074 \pm 0.001$ & h=24, Q.P. \\
\hline Jun 24 & 2210 & $191.7 \pm 5.5$ & 6 & 44.4 & $0.087 \pm 0.003$ & h=24, Q.P., first batch of 6 \\
\hline Jun 24 & 2210 & $194.3 \pm 4.0$ & 6 & 44.4 & $0.089 \pm 0.002$ & h=24, Q.P., other batch of 6 \\
\hline
\end{tabular}

Table II: time from inj. is the time since the bunch being measured was injected except for the last 2 rows where it is the time since the first bunch was injected (for the latter all of the bunches present were measured), Length is the full length of the bunch, \# is the number of bunches whose lengths were measured, Rf V from S. F. is the Rf voltage obtained from measuring the synchrotron frequency, bunch $\varepsilon$ is the $\varepsilon$ of a bunch with that length and Rf voltage found from Bbat. Q.P. stands for Quad mode pumping. Standard deviations are shown for the length measurements and $\varepsilon$ values.

\footnotetext{
${ }^{22}$ See K. Zeno," Overview and analysis of the 2016 Gold Run in the Booster and AGS", C-A/AP/523, August 2014. Pgs. 29-30. Note that I'm using 257 ns not 233 ns for the average length in the Feb 10 measurements (see BoosterAGS-EBIS Feb 102016 elog 1848 through 1859 entries). In regard to those I think it is fair to include the overshoot, for example the 1848 measurement looks fine to me. Although the Feb 11 measurement also ignores the overshoot, I didn't change its value. If I didn't ignore the overshoot it would be $286 \mathrm{~ns}$ long and the $\varepsilon$ would be $0.111 \mathrm{eV}-\mathrm{s} / \mathrm{n}$.
} 
Graphical representations at injection for both the $h=16$ and $h=24$ cases are shown in Figures 6 and 7 respectively (the bunch at Booster extraction is treated as if it were matched, which it isn't). In the $\mathrm{h}=16$ (unpumped) case, the value of $\Delta E_{f}$ is much smaller than the average value found for the $\mathrm{h}=24$ (pumped) case, so 2 curves are shown for the bunch after the foil, one using the $\Delta E_{f}$ found from the measurement of unpumped bunches (14.9 MeV, light blue) and the other using the average $\Delta E_{f}$ found for pumped bunches (24.6 MeV, gray). In either case, the entire injected bunch fits into the $\mathrm{h}=16$ bucket (red trace), but in the $\mathrm{h}=24$ case a significant portion of it lies outside the bucket.

After filamentation, one would expect the bucket to be filled in the $\mathrm{h}=24$ case, and depending on the actual value of $\Delta E_{f}$, one would expect it will be either filled or nearly filled in the $\mathrm{h}=16$ case. The $\mathrm{h}=24$ bucket area is only $0.091 \mathrm{eV}-\mathrm{s} / \mathrm{h}$ and in the $\mathrm{h}=16$ case it is $0.122 \mathrm{eV}-\mathrm{s} / \mathrm{n} .{ }^{23}$ In both cases the measured emittances are close to the respective bucket size, though slightly smaller.

As mentioned, one would expect more beam to fall outside the bucket in the $\mathrm{h}=24$ case, and so one should see a reduction in the efficiency of bunch transfer from the Booster to AGS. However, if there was a substantial amount of uncaptured beam surviving on the injection porch, a loss should be evident when the kicker fires on the next transfer when no beam is injected on that transfer, but this is not typically observed. ${ }^{24}$ It seems more likely that the amount of beam that falls outside the $\mathrm{h}=24$ bucket that survives in, or even gets to, the AGS is minimal. Even so, one might still expect a significant difference in the transfer efficiency between the $\mathrm{h}=16$ and 24 cases.

Calculating the bunch transfer efficiency is a somewhat complicated matter. During Run 16 using $\mathrm{h}=24$ the ratio of the bunch intensity on the AGS flattop divided by the bunch intensity at Booster extraction approached $50 \%$ and at that time the percentage of beam in the baby bunches was about $4 \% .{ }^{25}$ This means that just after the last transfer, assuming no loss from there to the flattop (which is close to being true), 52\% of the Booster bunch was in an AGS bunch. There is a slow loss on the injection porch, presumably mainly due to the quality of vacuum and that rate was measured to be $5.2 \% / \mathrm{sec}^{26}$

\footnotetext{
${ }^{23}$ The Rf voltage at injection is typically set to minimize bunch shape oscillations in order to match the injected bunch as well as possible. So, using the bucket areas for matched bunches does not seem unreasonable.

${ }^{24}$ See Booster-AGS-EBIS Apr 2720161812 through 1814 entries. This is when the conditions are optimized. It is not hard to intentionally make a capture loss in the AGS.

${ }^{25}$ See K. Zeno,"Overview and analysis of the 2016 Gold Run in the Booster and AGS", C-A/AP/523, August 2014. Pgs. 20-21.

${ }^{26}$ See Booster-AGS-EBIS Apr 272016 elog 1814 entry
} 
Bunch Shape before and After the Foil for an $\mathrm{h}=16$ bucket

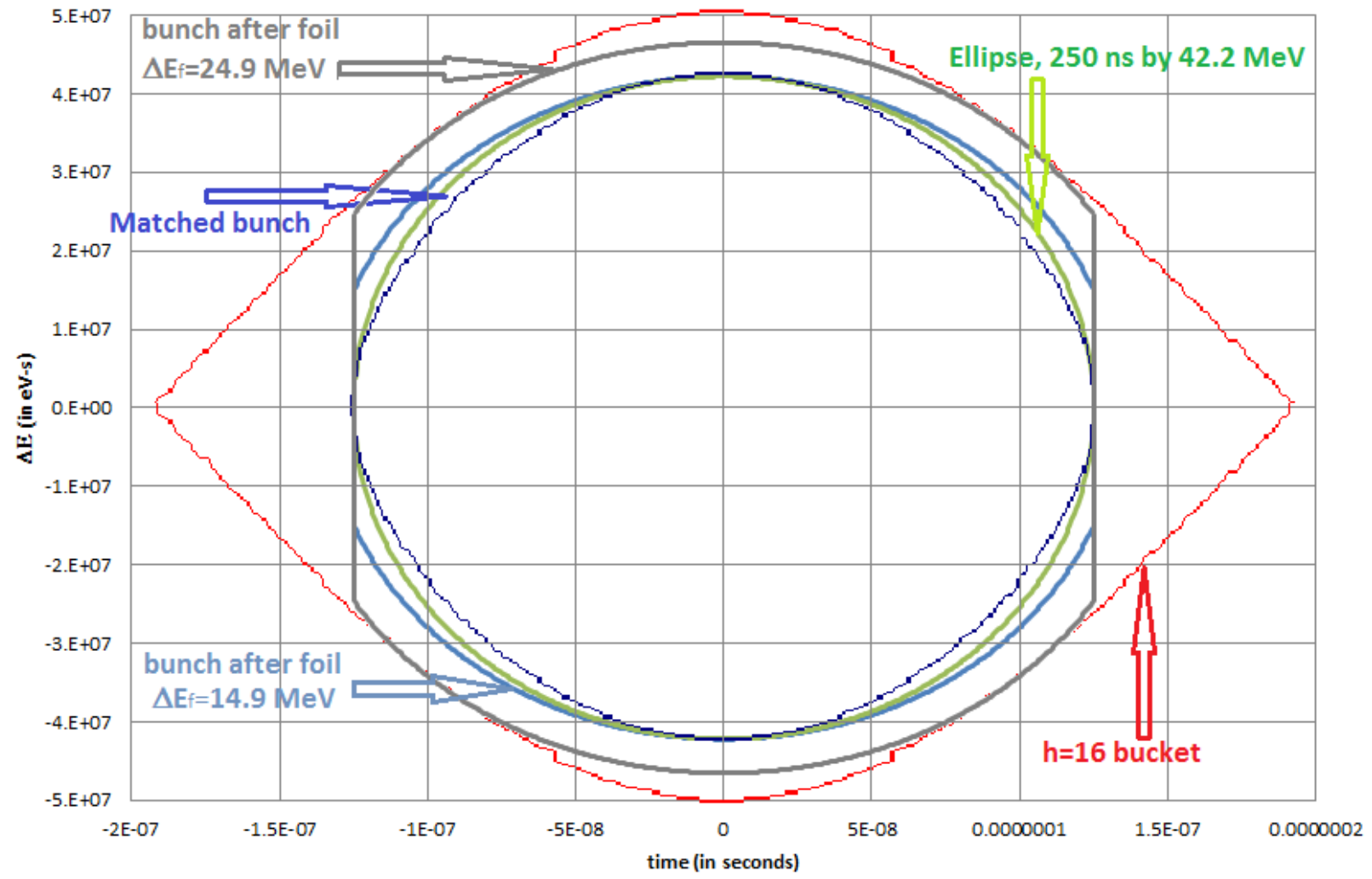

Figure 6: Injection into an h=16 AGS bucket of a 250 ns long bunch with an energy spread $\left(\Delta E_{a}\right)$ of 42.2 $\mathrm{MeV}$. The dark blue trace is a $250 \mathrm{~ns}$ long bunch with $\Delta E=\Delta E_{a}$ matched to the $\mathrm{h}=16$ bucket (red trace). The green trace is an ellipse with axes $250 \mathrm{~ns} / 2$ and $\Delta E_{a}$. The light blue trace is the bunch shape at injection calculated using the above $l$, the $\Delta E_{b}$ found for a merged bunch without pumping (39.5 MeV), the $\Delta E_{f}$ found for that case (14.9 MeV) and eq. (4). The gray trace is the bunch shape at injection calculated using the above $l$, the $\Delta E_{b}$ found for a merged bunch without pumping (39.5 MeV), the average $\Delta E_{f}$ found from the measurements with quad pumping (24.6 MeV) and eq. (4).

If one removes this loss from the efficiency calculation then the transfer efficiency goes from $52 \%$ to $55.1 \%$. The transfer efficiency is typically a few percent higher for $\mathrm{h}=16$, about $55 \%$ vs. $52 \%$ after the last transfer. ${ }^{27}$ Assuming the same loss rate, and considering that there are 8 transfers instead of 12, the efficiency after the slow loss is removed from the calculation becomes $57.1 \%$. Given that there is no discernable capture loss in either case it's tempting to attribute the reduction in efficiency from about 57 to $55 \%$ to greater losses due to momentum apertures during the transfer associated with the higher $\Delta p / p$ of the quad mode pumped $\mathrm{h}=24$ bunches. In the Tandem case there were 4 transfers and the transfer efficiency was about 58\%, again assuming the same loss rate and removing that loss from the calculation gives $58.9 \%{ }^{28}$

\footnotetext{
${ }^{27}$ See See K. Zeno," Overview and analysis of the 2016 Gold Run in the Booster and AGS", pg. 13.

${ }^{28}$ See P. Thieberger et al, "Improved gold ion stripping at 0.1 and $10 \mathrm{GeV} /$ nucleon for the Relativistic Heavy lon Collider" Phys. Rev. Spec Topics, 2008, pg. 011001-8
} 


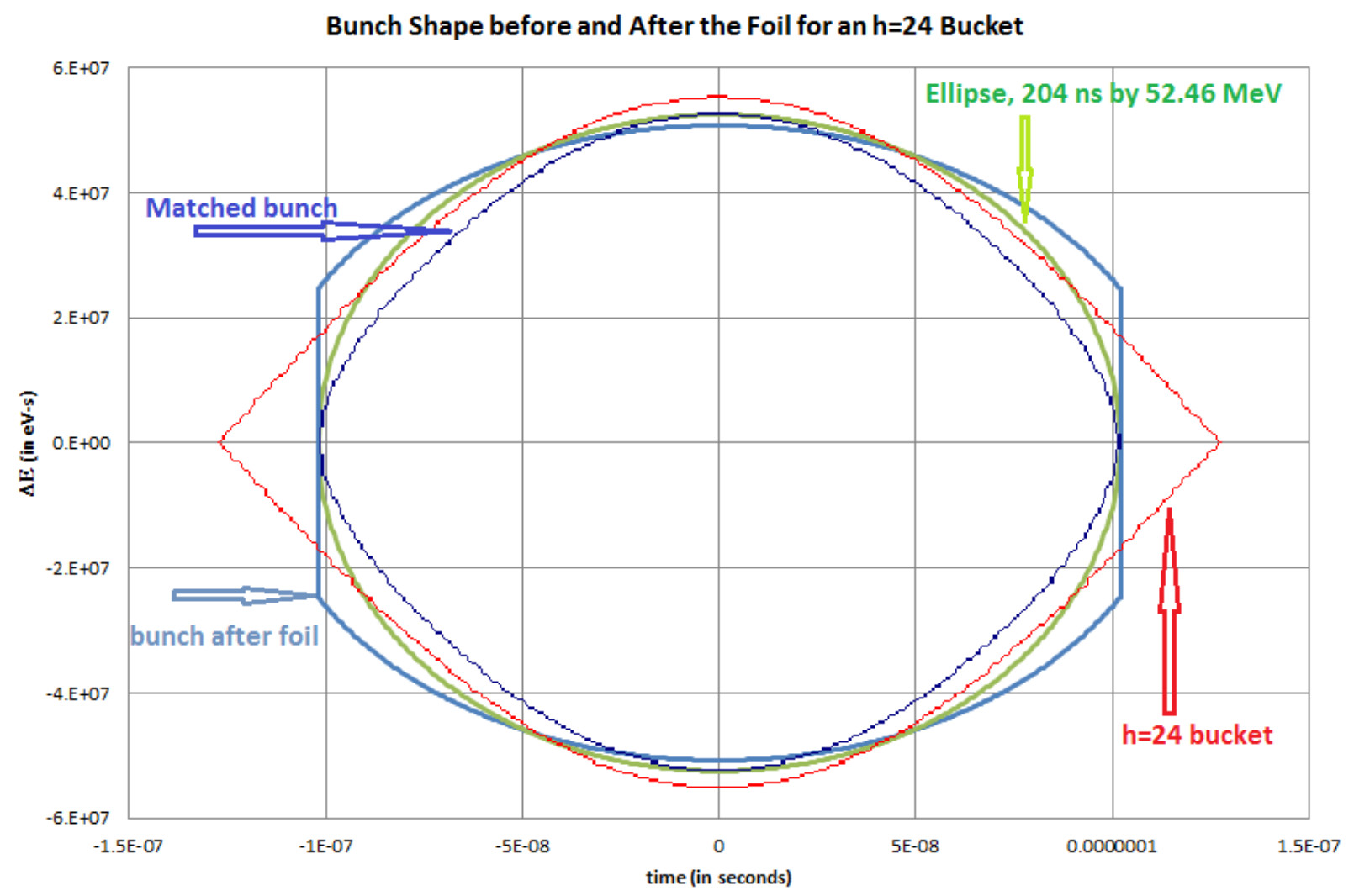

Figure 7: Injection into an h=24 AGS bucket of a quad mode pumped $204 \mathrm{~ns}$ long bunch with an energy spread $\left(\Delta E_{a}\right)$ of $52.46 \mathrm{MeV}$. The dark blue trace is a $204 \mathrm{~ns}$ long bunch with $\Delta E=\Delta E_{a}$ matched to the $\mathrm{h}=24$ bucket (red trace). The green trace is an ellipse with axes $204 \mathrm{~ns} / 2$ and $\Delta E_{a}$. The light blue trace is the bunch shape at injection calculated using the above $l$, the $\Delta E_{b}$ found for the quad pumped case (44.36 $\mathrm{MeV}$ ), the average value of $\Delta E_{f}$ found for that case (24.6 MeV) and eq. (4).

\section{Emittance Growth due to Energy Loss in the BtA Foil}

An issue also arises when injecting multiple bunches from one Booster cycle into the AGS through the BtA foil. ${ }^{29}$ In order for the injected bunches to be matched in phase to the AGS buckets, those buckets must have the same spacing as the ones in the Booster. Consequently, since the AGS circumference is 4 times that of the Booster, the AGS Rf harmonic must be 4 times that of the Booster, and there can be at most 4 sets of multiple bunches injected. Even in this case though, since the foil causes a net energy loss and the beam is far from relativistic ( $\beta=0.43$ ), the bunches that have emerged from the foil move more slowly than those that have not entered it yet and consequently, once in the AGS, they are spaced more closely together than they were in the Booster. Note that the AGS Rf frequency also needs to be lowered from what it would be if there was no energy loss so the bunches will be matched in energy to the AGS buckets, but that adjustment though necessary, does not fix this problem.

\footnotetext{
${ }^{29}$ L. Ahrens, et al, "Setup and Performance of the RHIC Injector Accelerators for the 2007 Run with Gold lons". Proceedings of PAC07, Albuquerque, New Mexico, USA , pg. 1862
} 
This was the case when Tandem was used to provide beam to RHIC. In that case, the revolution frequency at extraction was about $659 \mathrm{kHz}$, so the last of the 6 bunches extracted passes through the foil $(5 / 6)(1 / 659 \mathrm{kHz})=1.26 \mu$ s after the first bunch. The velocity difference before and after the foil, corresponding to the measured difference in energy of $2.5 \mathrm{MeV}$ (per nucleon) is $1.36 \mathrm{e} 6 \mathrm{~m} / \mathrm{s} .{ }^{30}$ So, after they have both passed through it, the $1^{\text {st }}$ and last bunch extracted are $(1.26 \mu \mathrm{s})(1.36 \mathrm{e} 6 \mathrm{~m} / \mathrm{s})=1.71 \mathrm{~m}$ closer than they would have been otherwise, and $1.71 \mathrm{~m}$ corresponds to $24^{*} 360^{\circ} *(1.71 \mathrm{~m} / 807 \mathrm{~m})=18.3^{\circ}$ of $\mathrm{h}=24$ phase (where $807 \mathrm{~m}$ is the AGS circumference). Dipole oscillations, which are thought to originate from this effect, are observed on the injection porch and typically the bunch does not filament out before the AGS merges. But even though they don't filament, because the oscillations are still present when the merges start, one might think that the effect on the $\varepsilon$ after the merge would be roughly the same as if they did.

Ideally, a phase adjustment is used to make the average bunch to bucket phase error over the 6 bunches as close to zero as possible, but regardless the pattern of dipole oscillations changed significantly from one transfer to the next. ${ }^{31}$ Figure 8 shows a case where the average phase error is not zero, but one of the bunches (the $2^{\text {nd }}$ from the left) is matched to its bucket. The bunch to bucket phase difference between adjacent bunches should be $\left(18.3^{\circ} / 5\right)$, so the bunch on the far right should be phase-mismatched by $4 *\left(18.3^{\circ} / 5\right)=14.6^{\circ}$. The $\mathrm{x}$-axis in the figure is the geometric phase around the ring and the amount of change in geometric phase corresponding to $\pm 14.6^{\circ}$ in an $\mathrm{h}=24$ bucket is $\pm 0.608^{\circ}$. Figure 9 shows the right most trace again, and 2 ways of measuring the amplitude of the phase oscillation. Using both methods I find that the total geometric phase oscillation is $1.17^{\circ}$, which is in reasonable agreement with the calculation $\left(2 * 0.608^{\circ}=1.22^{\circ}\right)$. The fact that the bunches do not filament out but continue to oscillate indicates that there are some stability issues, but if they did filament the 'effective' $\varepsilon$ would just become the actual one. ${ }^{32}$

In the case considered here, the length of the injected bunch is assumed to be 55 ns, but in the most extreme case, where the phase mismatch is $18.3^{\circ}$, the effective length obtained from making a bunch $\pm 18.3^{\circ}$ wider, would be $81 \mathrm{~ns}$, and with the Rf voltage matched to a bunch with $\Delta E_{a}=32 \mathrm{MeV}$, the 'effective' $\varepsilon$ would be $0.0294 \mathrm{eV}-\mathrm{s} / \mathrm{n} .{ }^{33}$ Similarly, in the case where the average phase mismatch is zero, the bunch with the largest phase mismatch $\left(9.15^{\circ}\right)$ has an effective length of $67.9 \mathrm{~ns}$ and effective $\varepsilon$ of $0.021 \mathrm{eV}-\mathrm{s} / \mathrm{n}$.

\footnotetext{
${ }^{30}$ See L. Ahrens, et al, "Setup and Performance of the RHIC Injector Accelerators for the 2007 Run with Gold lons". Proceedings of PAC07, Albuquerque, New Mexico, USA , pg. 1863, where it is indicated that the measured energy loss from the foil is $2.5 \mathrm{MeV}$.

${ }^{31}$ L. Ahrens, Booster-AGS Gold elog, April 20, 2011, Apr 260922 and 1925 entries,

32 Often in fact the dipole oscillations behave in a more complicated way. For example, their phases are not opposite on either side of the matched bunches (see L. Ahrens, Booster-AGS Gold elog, Apr 2620110925 entry).

${ }^{33}$ Note that in Figure 9 the length of the bunch is about $3^{\circ}$ of geometric phase and with $\beta=0.43$ this corresponds to a length of $52 \mathrm{~ns}$, which is close to $55 \mathrm{~ns}$.
} 




Figure 8: A mountain range display of the AGS wall current monitor signal showing the 6 bunches from one Booster transfer as they are injected and the subsequent dipole oscillations associated with the phase mismatch caused by the energy loss in the stripping foil. Time advances from the bottom to the top of the display. The $\mathrm{x}$-axis is the geometric phase around the AGS ring.

It may be that the 24:12:4 merge largely conserves $\varepsilon$ for 6 incoming bunches each of the same $\varepsilon$, but that is not true if they are different. Simulations where all the bunches have the same $\varepsilon$ coming into the merge indicate that the particles from each of the incoming bunches eventually more or less evenly fill the area occupied by the final bunch which is about 6 times larger than the $\varepsilon$ of a single pre-merged bunch. ${ }^{34}$ So, assuming all the beam is successfully merged, in the case where the bunches are of varying size one would think that the $\varepsilon$ of the final bunch would be 6 times the $\varepsilon$ of the largest bunch going into the merge. Now these bunches are still undergoing dipole oscillations and haven't filamented so their $\varepsilon$ has technically not increased, but it seems reasonable to think that the effect would be roughly the same as if they had. If so, the $\varepsilon$ of the merged bunch would tend to vary from $6 * 0.021=0.13$ up to $6 * 0.00294=0.18 \mathrm{eV}-\mathrm{s} / \mathrm{n}$ and the effective $\varepsilon$ coming into the AGS merges would be about 1.5 to 2.1 times larger than the injected $\varepsilon(0.084 \mathrm{eV}-\mathrm{s} / \mathrm{n})$.

\footnotetext{
${ }^{34}$ SeeC. Gardner, "Gold 631 merge and squeeze simulations in AGS"
} 


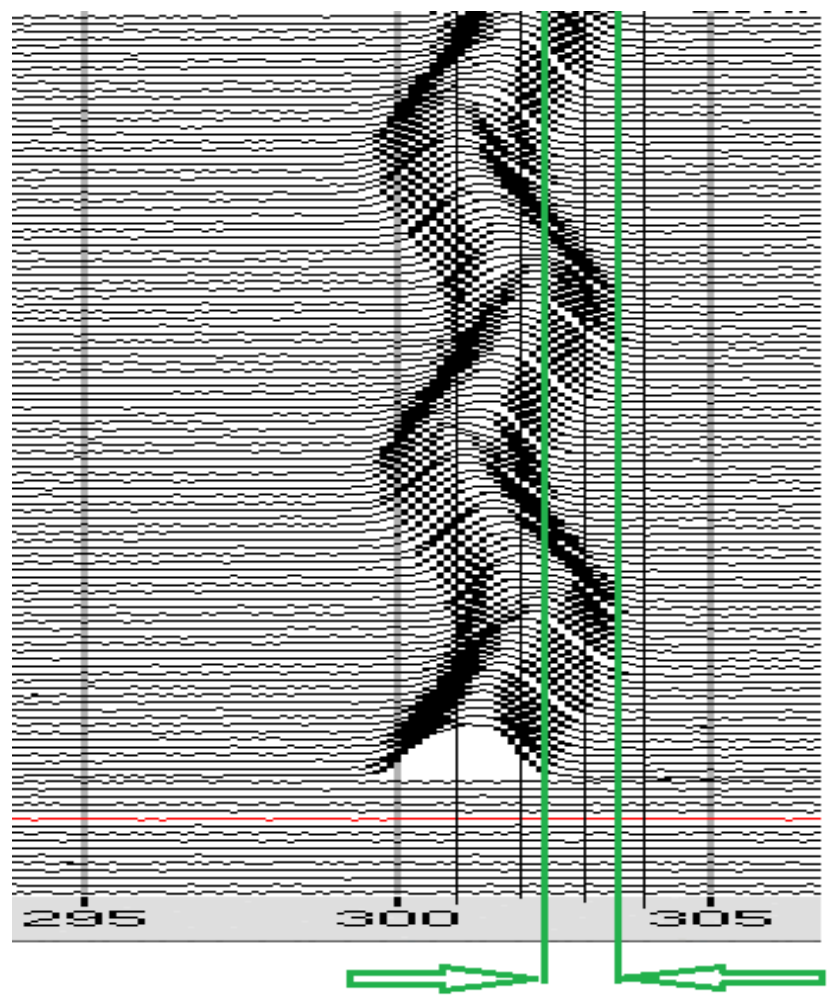

$1.17^{\circ}$

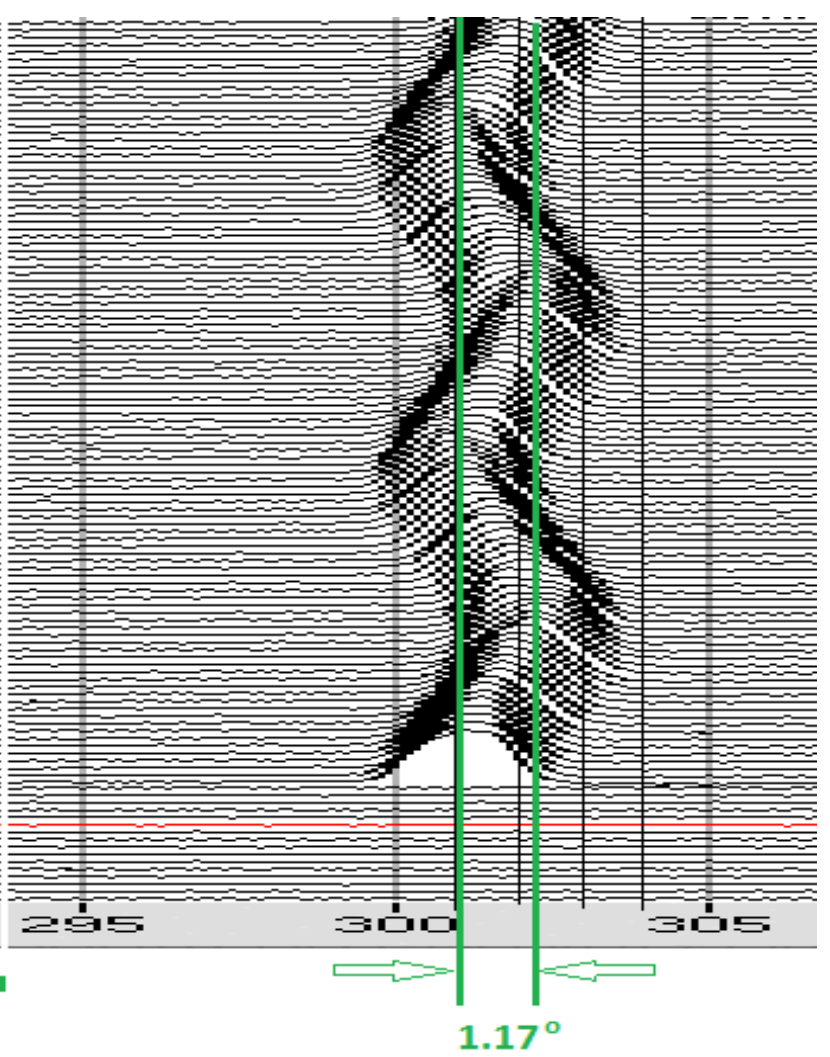

Figure 9: Two measurements of the amplitude of the dipole oscillations in terms of geometric phase around the AGS ring. The plots are of the rightmost bunch in Figure 8. In the left plot the 2 vertical green lines are placed at the right edge of the bunch when it is furthest to the left and furthest to the right and the difference in geometric phase is measured. In the right plot the 2 vertical lines are placed at the center of the bunch when it is furthest to the left and to the right and the difference in geometric phase is measured. Both methods give the same answer, $1.17^{\circ}$. In both plots the black vertical lines between $300^{\circ}$ and $305^{\circ}$ are spaced $1^{\circ}$ apart.

A set of $\varepsilon$ measurements was made in 2008 and just after the merges the $\varepsilon$ was $0.17 \pm 0.02$ $\mathrm{eV}$-s/n which is not inconsistent with the analysis above. ${ }^{35}$ Although there could be $\varepsilon$ growth from the merges themselves, much of the observed growth is not inconsistent with what could be accounted for by this phase mismatch. In which case, the total $\varepsilon$ growth due to the foil might be as much as a factor of $0.18 / 0.046$ or 3.9.

Although this effect was never observed for bunches using EBIS beam without a Booster merge because the AGS was never setup for them, there is no reason to think that it would not occur and an estimate for the effective $\varepsilon$ growth in that case can also be made. The injected

\footnotetext{
${ }^{35}$ E. Pozdeyev, "Longitudinal Beam Emittance", slide 5, upper left corner plot, 2008 RHIC Retreat. Note that these measurements indicate $\varepsilon$ growth from the merges, especially the 3 to 1 merge, but that is not necessarily inconsistent with the idea that the growth occurs from the persistent dipole oscillations going into the merges. Since the bunch does not filament out, its measured $\varepsilon$ would still not show the effect of the dipole oscillations until they are merged. It should also be noted that the author of this presentation only attributes about $20 \%$ growth due to the phase mismatch at injection and his $\varepsilon$ measurements were taken using a different method than the one used for measurements in this note and were also probably taken with a different wall current monitor.
} 
bunches would probably be more stable since they are less intense and larger, so they might filament. Also, the effect should not be as severe because the bunches are longer to begin with and they would be injected into $\mathrm{h}=16$ buckets. The last of the 4 bunches would pass through the foil $(3 / 4)(1 / 659 \mathrm{kHz})=1.14 \mu \mathrm{s}$ after the first and would be $(1.14 \mu \mathrm{s})(1.36 \mathrm{e} 6 \mathrm{~m} / \mathrm{s})=1.55 \mathrm{~m}$ closer to the first than it would've been otherwise which corresponds to $16^{*} 360^{\circ} *(1.55 \mathrm{~m} / 807 \mathrm{~m})=11.1^{\circ}$ of $\mathrm{h}=16$ phase. The new low level Rf system, implemented after the Tandem era, likely has much better bunch to bucket phase control, so it seems reasonable to treat the average bunch to bucket phase as zero.

The length of $\mathrm{h}=4$ Booster bunches at AGS injection was measured to be $79.1 \mathrm{~ns}$, in order to match their $\Delta E_{a}(36.7 \mathrm{MeV})$ the $\mathrm{h}=16 \mathrm{Rf}$ voltage needs to be $87.5 \mathrm{kV}$. The largest phase mismatch would be $5.5^{\circ}$, which in this case would cause the bunch's effective length to increase from 79.1 to 91.0 ns and its effective $\varepsilon_{\text {total }}$ would then be $4 * 0.0303 \mathrm{eV}-\mathrm{s} / \mathrm{n}=0.121 \mathrm{eV}-\mathrm{s} / \mathrm{n}$ (instead of $0.092 \mathrm{eV}-\mathrm{s} / \mathrm{n})$. So, starting out with an $\varepsilon_{\text {total }}$ of $0.068 \mathrm{eV}-\mathrm{s} / \mathrm{n}$ at Booster extraction, the (effective?) $\varepsilon$ coming into the AGS merges would be a factor of 1.78 larger than that instead of only 1.36 times larger without this effect.

In this analysis the incoming bunch has been treated as matched except in phase but as discussed earlier the shape of the incoming bunch does not match a standard $\mathrm{h}=16$ or 24 bucket (see Figure 1). This fact, although ignored here, might lead to even more $\varepsilon$ growth.

The effective $\varepsilon$ growth associated with energy loss passing through the foil only occurs because there are multiple bunches transferred in one Booster cycle. With only one bunch from the Booster and $\mathrm{h}=24$ at AGS injection the $\varepsilon$ going into the AGS merges appears to be only about 1.17 times larger than it is at Booster extraction. The Booster merge also likely contributes some growth although measurements suggest that it's relatively small, $(0.072 / 0.068)=1.06$. So, the total amount of $\varepsilon$ growth from this EBIS type setup is maybe about a factor of 1.25. However, in the Tandem case (or likely the EBIS case if no Booster merge were necessary) assuming no merge or acceleration losses, about $58 \%$ of the beam at Booster extraction would be expected to be in a bunch on the flattop, but for the 12:6:2 EBIS setup that efficiency is reduced to $50 \%$.

\section{Growth during AGS Acceleration for Different Emittances at the Beginning of the Ramp}

Another effect that contributes to the emittance on the AGS flattop is the growth that occurs during the acceleration ramp. The mechanism for this growth is unclear but main magnet ripple on the pulsed voltage bank is suspected.

In Run 17 a study was done with the bunches merged into one in the Booster but then longitudinally scraped before extraction. ${ }^{36}$ The bunch length of five of those bunches was

\footnotetext{
${ }^{36}$ See Booster-AGS-EBIS Apr 172017 elog, entries from 1712 to 1851
} 
measured after some filamentation but still at injection energy and their average length in $\mathrm{h}=12$ buckets was $161.8 \mathrm{~ns}$ with a $\sigma$ of $7.4 \mathrm{~ns}$. The Rf voltage found from the synchrotron frequency was $60.11 \mathrm{kV}$. Using Bbat, the $\varepsilon$ of these bunches was found to be $0.075 \pm 0.006 \mathrm{eV}-\mathrm{s} / \mathrm{n}$. This setup had no AGS merges and bunches were accelerated to the AGS flattop where their length was measured to be 10.67 ns with a $\sigma$ of $0.58 \mathrm{~ns}$. The Rf voltage found from the synchrotron frequency was $159.5 \mathrm{kV}$. Using Bbat, the $\varepsilon$ of these bunches was $0.102 \pm 0.011 \mathrm{eV}-\mathrm{s} / \mathrm{n}$. So, the $\varepsilon$ growth from injection to flattop is a factor of 1.36, but considering the standard deviations of the measurements it could be anywhere from 1.12 to $1.64 .{ }^{37}$ Measurements made in 2008 with the Tandem setup showed about a factor of 1.47 in $\varepsilon$ growth (about 0.017 to $0.025 \mathrm{eV}-\mathrm{s} / \mathrm{n}$ ). ${ }^{38}$

Measurements with the 12:6:2 EBIS setup indicate a factor of 1.44 in growth (initial $\varepsilon=0.51 \mathrm{eV}-\mathrm{s} / \mathrm{n}$ ) and measurements with the 8:4:2 EBIS setup indicate a factor of 1.45 in growth (initial $\varepsilon=0.415 \mathrm{eV}-\mathrm{s} / \mathrm{n}$ ). ${ }^{39}$ The $\varepsilon$ growth in all these cases is similar even though the initial emittance varies by a factor of 7 or so. Since this growth seems largely independent of the $\varepsilon$ at the beginning of the ramp (initial $\varepsilon$ ), the absolute amount of growth from injection energy to flattop just depends on the initial $\varepsilon$. In the earlier analyses it seemed natural to think that the proportional not the absolute growth would be the relevant parameter, but I find it somewhat surprising that this is also the case with growth during the ramp.

An implication of this is that since merging more Booster transfers into one AGS bunch creates a larger bunch at the beginning of the ramp it will also lead to more absolute growth during the ramp. In the case where the initial $\varepsilon$ was $0.075 \mathrm{eV}-\mathrm{s} / \mathrm{n}$ the absolute amount of growth was (0.102-0.075) eV-s/n=0.027 eV-s/n and in the case where 6 Booster transfers of merged bunches were merged into one it was $(0.75-0.51) \mathrm{eV}-\mathrm{s} / \mathrm{n}=0.24 \mathrm{eV}-\mathrm{s} / \mathrm{n}$, which is about 9 times as great.

\section{Summary}

The BtA stripping foil causes longitudinal $\varepsilon$ growth in two different ways. The first way is by increasing a bunch's energy spread via a mechanism known as energy straggling and the second is via energy loss.

The analysis indicates that the $\varepsilon$ growth from energy straggling is reduced from about a factor of 1.78 for unmerged Tandem bunches to 1.36 for unmerged EBIS bunches. The EBIS

\footnotetext{
${ }^{37}$ A similar, though perhaps not as accurate, measurement from Run 16 with even smaller $\varepsilon$ at injection $(0.051 \mathrm{eV}$ $\mathrm{s} / \mathrm{n}$ ) gave a factor of 1.47 in $\varepsilon$ growth. See pg. 34 of K. Zeno,"Overview and analysis of the 2016 Gold Run in the Booster and AGS", C-A/AP/523, August 2014.

${ }^{38}$ E. Pozdeyev, "Longitudinal Beam Emittance", Slide 5, upper left corner plot.

${ }^{39}$ See pg. 34 of K. Zeno," Overview and analysis of the 2016 Gold Run in the Booster and AGS", C-A/AP/523, August 2014.
} 
beam has less growth because its $\Delta E$ coming into the foil is larger since its total $\varepsilon$ before the foil is larger ( 0.068 vs. $0.046 \mathrm{eV}-\mathrm{s} / \mathrm{n})$ and since it is divided into 4 bunches instead of $6 .{ }^{40}$

Using an elliptical approximation the total $\varepsilon$ of (unmerged) Tandem beam after passing through the foil is estimated to be about $0.084 \mathrm{eV}-\mathrm{s} / \mathrm{n}$. A similar type estimate for the total $\varepsilon$ of unmerged EBIS beam is $0.092 \mathrm{eV}-\mathrm{s} / \mathrm{n}$. By merging the EBIS bunches into one in the Booster the $\varepsilon$ after passing through the foil is reduced to about $0.084 \mathrm{eV}-\mathrm{s} / \mathrm{n}$, the same value as the Tandem case, and the growth is about a factor of $1.17 .{ }^{41}$ But there is apparently some growth from the merge, about a factor of 1.06, so the total growth from using a merged setup is about a factor of 1.25. Although one might expect a further reduction from the use of quad mode pumping at Booster extraction, that reduction is not apparent.

Filamentation of merged bunches in the AGS also leads to $\varepsilon$ growth not only because the bunch has not filamented out from the Booster merge but also because the its shape after passing through the foil does not match an AGS Rf bucket. The growth due to filamentation depends on the shape and size of the Rf bucket, and it seems that an $\mathrm{h}=24 \mathrm{Rf}$ bucket leads to less growth due to filamentation than an $h=16$ bucket. In the $h=24$ case, the bunches from the Booster are also quad mode pumped to fit into $h=24$ buckets. In the $h=16$ case, the growth due to filamentation seems to be about a factor of $0.112 / 0.084=1.33$, but in the $h=24$ case the growth seems to be negligible.

This may help explain why the 12:6:2 merge setup, which employs $h=24$ buckets, had smaller $\varepsilon$ bunches going into the AGS merges than those in the 8:4:2 h=16 setup. Since the $\mathrm{h}=24$ bucket is considerably smaller than the $h=16$ bucket, one might expect a reduction in bunch transfer efficiency between injection into $\mathrm{h}=24$ and $\mathrm{h}=16$ buckets, and there is a reduction, but it appears to be only about $2 \%$, from 57 to $55 \%$.

It's expected that the growth in $\Delta E$ from the foil $\left(\Delta E_{f}\right)$ adds in quadrature with the $\Delta E$ of the incoming bunch to produce the $\Delta E$ of the bunch after the foil as in eq. (1). Values for $\Delta E_{f}$ were found for many cases (i.e.-merged with quad pumping, merged without pumping, unmerged from Tandem, and unmerged from EBIS). The values obtained with merged bunches vary quite a bit, but the average value of all 5 of those measurements, $22.6 \mathrm{MeV}$, is not very different from the values obtained for unmerged bunches (26.5 MeV for Tandem and

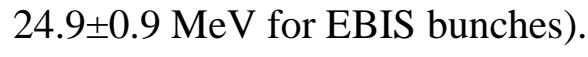

The $\varepsilon$ growth from energy loss only occurs with the transfer of multiple bunches from one Booster cycle. This was the standard operational mode with Tandem beam but has not been used with EBIS beam. The energy loss was measured to be $2.5 \mathrm{MeV} / \mathrm{n}$ with Tandem beam which had an extraction energy of 103.2 MeV and charge state of +31. The Booster extraction energy

\footnotetext{
${ }^{40} 0.068 \pm 0.005 \mathrm{eV}-\mathrm{s} / \mathrm{n}$ was the value obtained for the Booster extraction $\varepsilon$ in 2017 , but a measurement made in 2014 gave a significantly larger value, $0.084 \pm 0.005 \mathrm{eV}$-s/n.

${ }^{41}$ The merged bunch $\varepsilon$ estimate also uses the elliptical approximation, which is probably less valid in this case.
} 
for EBIS beam, $103.9 \mathrm{MeV}$, and charge state, +32, are nearly the same so the energy loss is assumed to be the same as well.

This growth occurs because the energy loss causes the bunches to be closer to each other than the centers of the AGS buckets they are injected into. This results in a phase mismatch at injection. It turns out that in the Tandem case this causes dipole oscillations that persist until the AGS merge. Normally one would expect the bunches to filament out before the time of the merge but apparently they do not because of some stability issue with the Tandem bunches. Regardless, the effect on the $\varepsilon$ after the merges is likely similar except that the growth appears to happen during the merges instead of before them.

This effect has been analyzed in some detail for the Tandem case and it is estimated that it lead to about a factor of 1.5 to 2.1 growth between injection and the end of the AGS merges. This estimate was compared with the measured growth and is not inconsistent with it as the measured $\varepsilon$ after the merges was about $0.17 \mathrm{eV}-\mathrm{s} / \mathrm{n}$ and the measured $\varepsilon$ at injection was about $0.084 \mathrm{eV}-\mathrm{s} / \mathrm{n}$.

An estimate of the amount of growth from this effect without a Booster bunch merge was made for EBIS beam with $h=4$ in the Booster and $h=16$ at AGS injection. In this case, a bunch would probably filament out before the AGS merges because it is likely to be more stable. Yet the growth from injection to after those merges would probably be similar whether it filamented out or not. But the growth would be less than it is for Tandem beam because the bunches are longer to begin with, they are injected into $\mathrm{h}=16$ buckets, and the bunch to bucket phase jitter is less with the Booster to AGS synchro that the new low level Rf system uses. The estimate for the growth in this case is a factor of 1.3 .

So, whereas in the Tandem case the growth from both effects of the foil on the $\varepsilon$ of a bunch after the AGS merges seems to have been about a factor of $1.78 * 1.5=2.7$ to $1.78 * 2.1=3.7$, in the EBIS case it would only be expected to be about a factor of $1.36^{*} 1.3$, or about 1.8 . This means that EBIS unmerged bunches after an 16:8:4 merge might be about $1.8 *(0.068 \mathrm{eV}-\mathrm{s} / \mathrm{n})=0.12 \mathrm{eV}-\mathrm{s} / \mathrm{n}$, which is smaller than what the $\varepsilon$ appears to have been for bunches of Tandem beam after the AGS merge (about $0.17 \mathrm{eV}-\mathrm{s} / \mathrm{n}$ ).

The use of merged Booster bunches together with $\mathrm{h}=24$ in the AGS seems to reduce the total growth from before the Booster merge to after the AGS merges to about a factor of 1.25, although there is some reduction in efficiency. If the EBIS injected intensity was sufficient to satisfy RHIC without a bunch merge in the Booster and by only merging the beam in 1 Booster cycle into one AGS bunch, as much as $58 \%$ of the beam at Booster extraction might be expected to be in one AGS bunch at extraction, with the present 12:6:2 setup it is at best $50 \%$.

Another contributing factor to $\varepsilon$ growth, which is only indirectly related to the foil, is the growth that occurs during the AGS acceleration ramp. Measurements over a large range of 
emittances at the beginning of the ramp indicate that the $\varepsilon$ grows by a factor of about 1.45 during the ramp regardless of the $\varepsilon$ at the beginning of it. This means that the smaller the $\varepsilon$ at the beginning of the ramp the less absolute growth occurs during the ramp. In the case considered with the smallest $\varepsilon$ at the beginning of the ramp, $0.075 \mathrm{eV}-\mathrm{s} / \mathrm{n}$, the absolute growth up the ramp was $0.027 \mathrm{eV}-\mathrm{s} / \mathrm{n}$. In the case considered with the largest $\varepsilon$ at the beginning of the ramp, $0.51 \mathrm{eV}-\mathrm{s} / \mathrm{n}$, the absolute growth was about $0.24 \mathrm{eV}-\mathrm{s} / \mathrm{n}$. 
